\title{
Primary Care Provider Knowledge and Practice in Risk Assessment for Early Age Onset Colorectal Cancer: Opportunities for Improvement
}

\author{
Anjali Parekh', Camille J. Hochheimer ${ }^{2}$, Jeannine M. Espinoza ${ }^{3}$, Jordan J. Karlitz ${ }^{4}$, Carmen L. Lewis ${ }^{4}$, \\ Sachin Wani ${ }^{5}$, Swati G. Patel ${ }^{4}$ \\ ${ }^{1}$ Division of Gastroenterology \& Hepatology, University of Colorado Anschutz Medical Center, ${ }^{2}$ Center for Innovative Design \\ \& Analysis, University of Colorado School of Public Health, Aurora, CO, ${ }^{3}$ Division of Gastroenterology \& Hepatology, Denver \\ Health Hospital Authority, Denver, CO, ${ }^{4}$ Division of General Internal Medicine, University of Colorado Anschutz Medical \\ Center, ${ }^{5}$ Rocky Mountain Regional Veterans Affairs Medical Center, Aurora, CO, USA
}

Colorectal cancer (CRC) incidence and mortality are rising in individuals under age 50, termed early age onset (EAO) CRC. Lower endoscopy is recommended for all patients with unexplained iron deficiency anemia (IDA) or hematochezia to assess the EAOCRC. For those without symptoms, professional societies recommend decreasing the age to start screening from 50 to 45 . Primary care provider (PCP) knowledge and practices around EAO-CRC risk assessment and screening are unknown. We conducted a survey study in May, 2020 of multi-specialty PCPs from three large medical systems to assess PCP knowledge, risk stratification practices and barriers/facilitators they face to offer CRC screening in patients $<50$. We conducted univariate analysis to assess factors associated with knowledge and diagnostic practices. Response rate was $27.7 \%$ (196/708). Although $77.6 \%$ of respondents were aware that EAO-CRC incidence is increasing, only $42.9 \%$ knew that EAO-CRC mortality is also increasing. Of note, $91.8 \%$ recommend starting average risk screening at age 50. For 40- to 49-year-old patients present with unexplained IDA or hematochezia, $71.9 \%$ and $50.5 \%$ of respondents, respectively, recommend a diagnostic colonoscopy. Trainees were less likely to be aware of rising EAO-CRC mortality (odds ratio, $0.42 ; 95 \% \mathrm{Cl}, 0.21$ to 0.82 ) and non-internal medicine providers were less likely to recommend $\mathrm{CRC}$ screening in those with a first-degree relative with $\mathrm{CRC}$ (odds ratio, $0.82 ; 95 \% \mathrm{Cl}, 0.72$ to 0.93 ). Ongoing education efforts will be required to improve recognition and management of high-risk symptoms, particularly among non-internists and trainees.

Key Words Colorectal neoplasms, Early detection of cancer, Gastrointestinal hemorrhage, Iron deficiencies, Primary health care

\section{INTRODUCTION}

The incidence and mortality of colorectal cancer (CRC) in patients under the age of 50 , or early age onset (EAO) CRC, are rising; $11 \%$ of all colon cancers and $15 \%$ of all rectal cancers in the United States (US) are diagnosed in individuals under age 50 [1-3]. EAO-CRC patients commonly present with symptoms, such as hematochezia and iron deficiency anemia (IDA) [4,5]; thus professional societies currently recommend lower endoscopic evaluation for patients with unexplained symptoms $[6,7]$. Unfortunately, there is a significant delay from the symptom onset to diagnosis in patients with EAO-CRC (152 to 217 days) compared to those diagnosed with CRC over age 50 (30 to 87 days) [4]. However, the extent to which this delay is patient- versus provider-mediated is unclear.

CRC screening in the US has historically been recommended starting at age 50, though professional societies recommended average-risk screening in African Americans to start at age 45 in 2008 due to higher incidence and CRC-related mortality in this group $[6,8]$ In response to the overall increasing burden of EAO-CRC in the US, the American Cancer Society (ACS) in 2018 [9] and most recently the United States Preventative Services Task Force (USPSTF) in 2021 [10] and the United States Multi-Society Task Force (US-MSTF) on CRC [11] have decreased the age to begin

Received October 25, 2021, Revised December 9, 2021, Accepted December 22, 2021

Correspondence to Swati G. Patel, E-mail: Swati.Patel@cuanschutz.edu, https://orcid.org/0000-0002-1035-4924

Check for updates 
average-risk CRC screening from 50 to 45 , as have done other societies [12,13]. Primary care providers (PCPs) are responsible for symptom assessment and CRC screening recommendations, but there is limited data on PCP knowledge about EAO-CRC or PCP practices in CRC risk assessment in patients under the age of 50 .

The aims of our study were to assess PCPs' knowledge of EAO-CRC epidemiology, PCP screening and risk-stratification practices (both in terms of symptoms and cancer family history) in individuals under age 50, and PCPs' perceptions of barriers and facilitators to CRC screening in patients under age 50 .

\section{METHODS}

\section{Design \& participants}

This was a multi-center survey study where PCPs affiliated with an academic medical center, a Veterans Affairs (VA) medical center, and a safety net system in Colorado were invited to participate. Each system has a hospital-based primary care practice and multiple ambulatory locations around the state (the academic, VA and safety net system have four, nine and two ambulatory clinics, respectively). This study was reviewed and approved by the Colorado Multiple Institutions Review Board, Protocol 19-1855.

Eligible participants were identified using hospital system email databases and included non-trainees and trainees in internal medicine (IM), family medicine, medicine/pediatrics (providers who are double-boarded in IM and Pediatrics and care for both populations), and gynecology (a specialty which provides primary care to women, including cancer prevention care). Medical doctors (MDs), doctors of osteopathic medicine (DOs), nurse practitioners and physician's assistants were included, since all of these individuals provide direct patient care. Specialty, year in training (if applicable), degree, hospital affiliation, and sex were obtained from public hospital records for all eligible participants.

\section{Survey instrument design \& distribution}

The survey was designed to capture three main domains: (1) knowledge of EAO-CRC epidemiology, (2) colorectal screening and diagnostic practices in patients under age 50, and (3) barriers and facilitators to screening in this population. Content validity (the extent to which our instrument measured the intended constructs) [14] of the survey was assessed by four national experts in CRC prevention and EAO-CRC. To assess readability, clarity, and layout in different formats (desktop vs. mobile device), pre-testing was performed with a focus group of nine gastroenterologists and three PCPs. Pilot testing was performed by seventeen clinicians from the three practice environments.

The final survey tool is a 44-item questionnaire which also included self-reported demographics (Supplementary Materials) and was implemented in Research Electronic Data Cap- ture. All eligible participants were sent a unique email survey link in May 2020. Weekly reminders were sent to non- and partial responders for three consecutive weeks.

We "a priori" selected four survey questions to assess provider characteristics that may be associated with EAO-CRC knowledge and guideline-based CRC risk assessment $[6,7]$ : (1) knowledge of EAO-CRC mortality trends, (2) referral for CRC screening for those ages 40 to 49 with a first-degree relative (FDR) diagnosed with $\mathrm{CRC}$ under age 60 , (3) referral for colonoscopy in 40- to 49-year-old patients with unex-

Table 1. Baseline characteristics

\begin{tabular}{|c|c|}
\hline Participant characteristic & $\begin{array}{c}\text { Value } \\
(N=196)\end{array}$ \\
\hline \multicolumn{2}{|l|}{ Provider sex } \\
\hline Male & $68(34.7)$ \\
\hline Female & $100(51.0)$ \\
\hline Missing & $28(14.3)$ \\
\hline \multicolumn{2}{|l|}{ Specialty } \\
\hline Internal medicine & $98(50.0)$ \\
\hline Family medicine & $53(27.0)$ \\
\hline Other $^{\mathrm{a}}$ & $20(10.2)$ \\
\hline Missing & $25(12.8)$ \\
\hline \multicolumn{2}{|l|}{ Training status } \\
\hline Trainee & $71(36.2)$ \\
\hline Independent provider & $74(37.8)$ \\
\hline Missing & $51(26.0)$ \\
\hline \multicolumn{2}{|l|}{ Degree } \\
\hline MD/DO & $146(74.4)$ \\
\hline Other & $25(12.8)$ \\
\hline Missing & $25(12.8)$ \\
\hline Patient diagnosed under age 50 & $43(21.9)$ \\
\hline Someone in personal life diagnosed under age 50 & $58(29.6)$ \\
\hline \multicolumn{2}{|l|}{ Patients diagnosed between age 30 and 50} \\
\hline $50 \%$ or less & $137(69.9)$ \\
\hline More than $50 \%$ & $34(17.3)$ \\
\hline Missing/“I don’t know” & $25(12.8)$ \\
\hline \multicolumn{2}{|l|}{ Patients with Medicare } \\
\hline $50 \%$ or less & $114(58.2)$ \\
\hline More than $50 \%$ & $23(11.7)$ \\
\hline Missing/“I don’t know” & $59(30.1)$ \\
\hline \multicolumn{2}{|l|}{ Patients with Medicaid } \\
\hline $50 \%$ or less & $80(40.8)$ \\
\hline More than $50 \%$ & $55(28.1)$ \\
\hline Missing/“I don’t know” & $61(31.1)$ \\
\hline \multicolumn{2}{|l|}{ Patients with private insurance } \\
\hline $50 \%$ or less & $110(56.1)$ \\
\hline More than $50 \%$ & $31(15.8)$ \\
\hline Missing/“I don’t know” & $55(28.1)$ \\
\hline \multicolumn{2}{|l|}{ Patients with VA benefits } \\
\hline $50 \%$ or less & $108(55.1)$ \\
\hline More than $50 \%$ & $48(24.5)$ \\
\hline Missing/“I don’t know” & $40(20.4)$ \\
\hline
\end{tabular}

Values are presented as number (\%). MD, medical doctor; DO, doctor of osteopathy; VA, veterans affairs. ${ }^{2}$ Medicine/pediatrics, pediatrics, obstetrics \& gynecology. 
plained hematochezia and (4) referral for colonoscopy in 40to 49-year-old patients with unexplained IDA.

\section{Statistical analysis}

Chi-square tests were used to compare responders and non-responders. Univariable models were built using logistic regression, accounting for clustering within the three practice environments. We reported odds ratios (ORs) and 95\% Cls, where a $\mathrm{Cl}$ that does not include one is considered significant. The univariable analysis was restricted to those survey respondents who provided complete survey responses. These analyses were completed using Stata/SE 17.0 and all results were evaluated at the $5 \%$ level.

\section{RESULTS}

\section{Baseline characteristics}

There was a $27.7 \%$ response rate $(196 / 708)$. The majority of responders were MDs or DOs $(74.4 \%)$, female $(51.0 \%)$, and IM (50.0\%). Of responders, $37.8 \%$ were non-trainees and $36.2 \%$ were trainees (Table 1 ). The majority of respondents were affiliated with the academic system (52.5\%), $24.0 \%$ were from the safety net system and $23.5 \%$ from the VA system. When comparing responders to non-responders, there was a higher proportion of trainees (39.8\% vs. $26.0 \%, P<$ 0.001 ) who responded to the survey but there was no significant difference based on degree, sex or specialty (Table S1).

\section{Knowledge of EAO-CRC epidemiology}

Although $77.6 \%$ of respondents were aware that EAO-CRC incidence is increasing in the US, only $42.9 \%$ knew that mortality is also increasing. The majority $(76.5 \%)$ are aware that $11 \%$ of all colon cancers in the US are diagnosed in patients under age 50 (multiple choice selection of $0 \%$ to $20 \%$ ); however only $56.6 \%$ were aware that $15 \%$ of all rectal cancers occur in patients under age 50 (multiple choice selection of $0 \%$ to $20 \%$ ).

\section{PCP screening practices}

A minority (19.4\%) discuss CRC screening when they first meet a patient, whereas most $(66.8 \%)$ report first talking about CRC screening at a specific age (40: 3.8\%, 45: 9.9\%, 45 to $49: 8.4 \%, 50: 72.5 \%)$. The majority of respondents (91.8\%) recommend starting CRC screening at age 50 in average risk individuals, and in May 2020 when the survey was administered, $86.7 \%$ would 'never' or 'rarely' offer CRC screening to average risk patients aged 40 to 49 without family history of CRC (Fig. 1). When asked specifically about African American patients, $14.3 \%$ reported that they would offer colorectal screening to patients under age 50, whereas $27.0 \%$ reported that they did not know or were neutral and $51.5 \%$ reported they would not. The majority of responders $(84.2 \%)$ identified the USPSTF guidelines as most impactful in their individual screening practices.

\section{Identification of high-risk subgroups}

The majority of responders $(69.9 \%)$ recommend CRC screening most of the time or always in individuals 40 to 49 who have an FDR diagnosed with CRC under age 60 (Fig. 1). For 40 to 49 year-olds with IDA, hematochezia, or change in bowel patterns, $71.9 \%, 50.5 \%$ and $25.5 \%$ of respondents, respectively, recommend a diagnostic colonoscopy most of the time or always. When asked more broadly about "any" diagnostic testing in patients under age 50 with symptoms, $73.0 \%, 56.1 \%$ and $68.9 \%$ recommend evaluation for those with IDA, change in bowel patterns and hematochezia, respectively.

\section{Barriers \& facilitators to screening}

The majority reported that their assessment of a patient's risk for CRC $(82.1 \%)$, guidelines $(79.6 \%)$, screening standards at their practice $(71.4 \%)$, and their perceived effectiveness of screening $(61.2 \%)$ were 'important' or 'very important' in their decision to offer CRC screening to patients under age 50 . Fewer respondents reported that patients' request for screening (53.6\%), time available to discuss screening at clinic visits $(51.0 \%)$, and insurance coverage $(31.1 \%)$ were 'important'

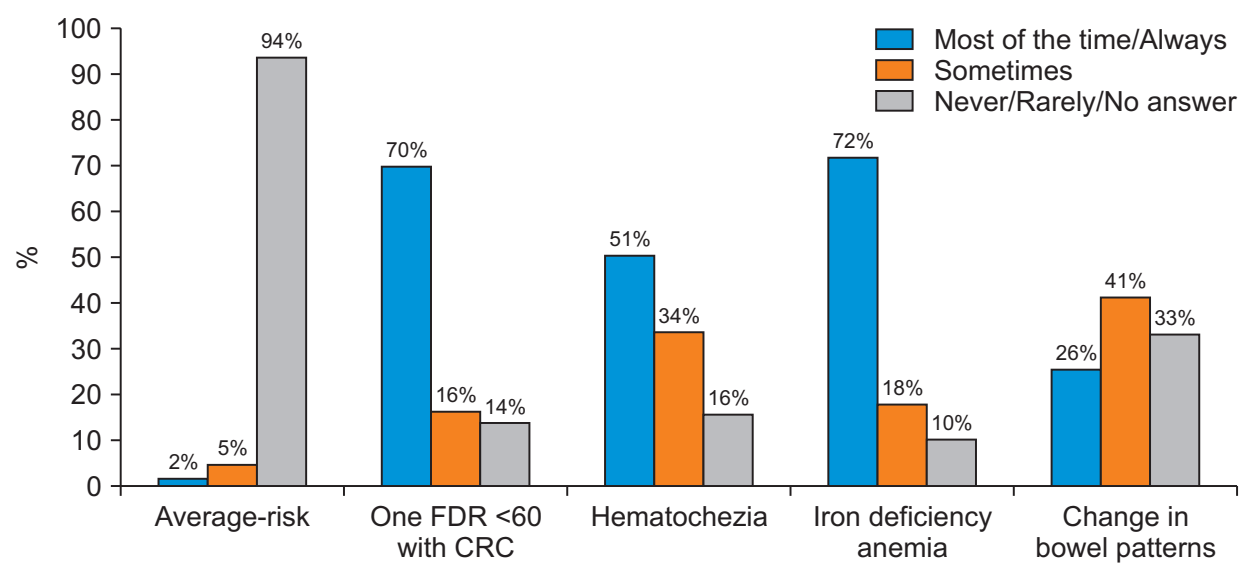

Figure 1. Scenarios in which primary care providers recommend colorectal cancer screening or diagnostic testing in patients between ages 40 and 49 . FDR, firstdegree relative; CRC, colorectal cancer. 
or 'very important' in their decision to offer CRC screening to patients under age 50 . It is noticeable that $64.3 \%$ of responders said convincing evidence of benefit would very much increase their likelihood of referring average risk patients under the age of 50 for CRC screening.

\section{Factors associated with gaps in knowledge \& practice}

Univariable analyses were limited to the 171 respondents who provided complete baseline characteristics. Compared to IM, family medicine providers were less likely to offer $\mathrm{CRC}$ screening to 40 to 49 year olds with an FDR with CRC diagnosed under age 60 (OR, $0.82 ; 95 \% \mathrm{Cl}, 0.72$ to 0.93 ) and less than half as likely to refer a patient age 40 to 49 for a diagnostic colonoscopy to evaluate IDA (OR, 0.46; 95\% $\mathrm{Cl}, 0.23$ to 0.91$)$. Trainees were less than half as likely to be aware that EAO-CRC mortality is increasing (OR, 0.42; 95\% $\mathrm{Cl}, 0.21$ to 0.82 ) and less likely to refer patients aged 40 to 49 for screening based on family history $(\mathrm{OR}, 0.44 ; 95 \% \mathrm{Cl}, 0.23$ to 0.83 ) or diagnostic colonoscopy for evaluation of hematochezia (OR, $0.41 ; 95 \% \mathrm{Cl}, 0.20$ to 0.84 ) (Table 2).

\section{DISCUSSION}

In response to the increasing incidence and mortality associated with $\mathrm{CRC}$ in individuals under age 50 , multiple professional societies, including the ACS [9], the USPSTF [10], the US-MSTF [11], the American College of Gastroenterology [12] and the National Comprehensive Cancer Network [13] have recommended changing the age to start CRC screening in average-risk individuals from 50 to 45 . There is minimal data about PCP approach to CRC risk assessment in individuals under age 50 . Our study shows that PCPs have variable knowledge of EAO-CRC epidemiology and most do not consider screening in average-risk patients under age 50 . As of May 2020, the majority of PCPs (92\%) recommend starting average risk CRC screening at age 50 , though $75 \%$ identified USPSTF guidelines as the most impactful in their screening practices. Thus, with the publication of a Grade B recommendation from the USPSTF in May 2021 to expand average risk screening to 45 to 49 year-olds [10], our data suggests that PCP screening practices will align with new guidelines to start CRC screening at age 45.

Multiple professional societies recommend CRC screening starting at age 40 (or 10 years prior to CRC diagnosis, whichever comes first) in those with an FDR diagnosed with CRC at any age $[6,12,13]$ and diagnostic lower endoscopy for young patients with unexplained symptoms $[6,7,12]$. The family history of colorectal neoplasia is an established risk factor for EAO-CRC $[15,16]$ and the majority of EAO-CRCs present with symptoms (most commonly hematochezia and IDA) $[4,5]$. Unfortunately, a large proportion of PCPs do not routinely recommend $\mathrm{CRC}$ screening or diagnostic colonoscopy in established high-risk groups. In our study, only $70 \%$ of PCPs routinely recommend CRC screening in 40 to 49 year-olds with an FDR with CRC. Only $73 \%$ and $69 \%$ recommend any diagnostic evaluation of IDA and hematochezia, respectively. Our results suggest that provider practices may contribute to the significant delays observed between symptom onset and CRC diagnosis in patients under the age of 50. These results highlight important targets for continuing education and implementation of clinical pathways to promote guideline-based screening based on family history, and diagnostic evaluation for symptomatic patients.

Our study has also identified subgroups of PCPs who may benefit from targeted interventions to improve screening and

Table 2. Characteristics associated with primary care provider knowledge, screening and diagnostic colonoscopy referral practices for patients aged between 40 and 49

\begin{tabular}{|c|c|c|c|c|}
\hline Baseline characteristic & $\begin{array}{l}\text { Knowledge of } \\
\text { rising mortality }\end{array}$ & FDR with $\mathrm{CRC}^{\mathrm{a}}$ & Hematochezia $^{a}$ & $\begin{array}{l}\text { Iron deficiency } \\
\text { anemia }^{a}\end{array}$ \\
\hline Female vs. male & $1.12(0.62$ to 2.03$)$ & $1.60(0.72$ to 3.58$)$ & $0.75(0.47$ to 1.18$)$ & 1.34 (0.59 to 3.03$)$ \\
\hline Family medicine vs. internal medicine & 1.09 (0.88 to 1.36$)$ & $0.82(0.72$ to 0.93$)$ & $1.38(0.97$ to 1.97$)$ & $0.46(0.23$ to 0.91$)$ \\
\hline Other ${ }^{b}$ vs. internal medicine & $2.14(0.56$ to 8.10$)$ & $0.68(0.28$ to 1.67$)$ & $1.47(0.51$ to 4.27$)$ & $0.79(0.16$ to 3.86$)$ \\
\hline Trainee vs. independent provider & $0.42(0.21$ to 0.82$)$ & $0.44(0.23$ to 0.83$)$ & $0.41(0.20$ to 0.84$)$ & $0.34(0.11$ to 1.05$)$ \\
\hline MD/DO vs. other provider & 0.69 (0.30 to 1.58$)$ & $1.22(0.47$ to 3.19$)$ & $0.50(0.38$ to 0.67$)$ & 2.46 (2.18 to 2.77$)$ \\
\hline Patient diagnosed with $\mathrm{CRC}$ age $<50$ & $1.66(0.44$ to 6.25$)$ & $1.60(0.45$ to 5.74$)$ & $1.41(0.73$ to 2.73$)$ & $0.85(0.17$ to 4.25$)$ \\
\hline Personal contact diagnosed with $\mathrm{CRC}$ age $<50$ & $1.43(0.37$ to 5.51$)$ & 0.91 (0.66 to 1.26$)$ & $2.29(0.81$ to 6.44$)$ & $3.17(1.59$ to 6.32$)$ \\
\hline More than $50 \%$ patients between ages 30 to 50 & 0.86 (0.73 to 1.02$)$ & $1.68(0.93$ to 3.03$)$ & 1.09 (0.94 to 1.27$)$ & 2.31 (1.22 to 4.38$)$ \\
\hline More than $50 \%$ patients have Medicare & 1.15 (0.64 to 2.08$)$ & 0.79 (0.38 to 1.62$)$ & $1.73(0.81$ to 3.71$)$ & $0.77(0.48$ to 1.23$)$ \\
\hline More than $50 \%$ patients have Medicaid & $0.50(0.27$ to 0.95$)$ & $2.10(1.07$ to 4.13$)$ & $0.71(0.31$ to 1.58$)$ & $0.94(0.41$ to 2.14$)$ \\
\hline More than $50 \%$ patients have private insurance & $1.26(0.46$ to 3.47$)$ & $1.13(0.52$ to 2.47$)$ & $1.03(0.67$ to 1.59$)$ & $0.82(0.72$ to 0.93$)$ \\
\hline More than $50 \%$ patients have VA benefits & 1.56 (0.92 to 2.63$)$ & $1.06(0.82$ to 1.38$)$ & $2.87(1.89$ to 4.38$)$ & $0.68(0.43$ to 1.07$)$ \\
\hline
\end{tabular}

Values are presented as odds ratio $(95 \% \mathrm{Cl})$. FDR, first degree relative; CRC, colorectal cancer; MD, medical doctor; DO, doctor of

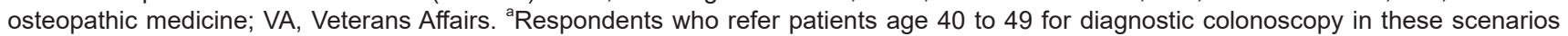
'most of the time' or 'always'. 'Medicine/pediatrics, pediatrics, obstetrics \& gynecology. Analysis included participants who provided complete responses to all self-reported baseline characteristics $(\mathrm{N}=171)$. 
diagnostic evaluation. IM specialty, non-trainees, and MD/DO degree were identified as factors associated with knowledge of EAO-CRC epidemiology and guideline concordant practices in 40 to 49 year-olds with a family history of CRC or symptoms. Thus, interventions may be most beneficial in non-IM specialties, advanced practice providers, and trainees in particular, as a call to action to expand education on EAO-CRC at the medical school and residency levels. Reinforcing these topics early in training may help to reverse the knowledge and practice gaps observed in non-trainees. It is not surprising that PCPs who know someone who has been diagnosed with $\mathrm{CRC}$ under age 50 are more likely to refer patients with IDA for diagnostic testing.

Our study has several limitations. Though a response rate of nearly $30 \%$ is robust among busy PCPs in the midst of a global pandemic and physician-based surveys may be less affected by non-response bias compared with other surveys due to higher homogeneity of knowledge, training, attitudes and behaviors than other groups [17], some survey responses were incomplete, which precluded multivariable analysis of factors associated with knowledge and practices. We did minimize sampling bias by surveying PCPs at a tertiary care center, a safety net hospital, and a VA medical center, but we acknowledge that all of these hospitals are within a single state and results may not be generalizable to all PCPs in the US. In particular, a possible reason why the majority of PCPs did not recommend average-risk screening in those under age 50 is because the state of Colorado only acknowledges USPSTF recommendations for insurance coverage, which were not updated to include patients younger than 50 years old until May, 2021 (unlike other states that also acknowledge the 2018 ACS guidelines). Results may have been different in states that dictate coverage based on both USPSTF guidelines and ACS guidelines.

In conclusion, our study highlights knowledge gaps among PCPs of the changing epidemiology of CRC and evidence-based risk assessment of those with red flag symptoms. Important future work will include assessing the impact of recently published USPSTF guidelines on PCP screening practices; however, ongoing education efforts will be required to improve recognition and management of high-risk symptoms, particularly among non-IM primary care specialties and trainees.

\section{ACKNOWLEDGMENTS}

Colorado Clinical \& Translational Sciences Institute (CCTSI) with the Development and Informatics Service Center (DISC) grant support (NIH/NCRR Colorado CTSI Grant Number UL1 RR025780). This project was presented as an oral abstract at the Digestive Diseases Week 2021 Conference.

\section{CONFLICTS OF INTEREST}

AP: None, CJH: None, JE: None, JJK: Advisor for Exact Sciences, Equity position and Chief Medical Officer for Gastro Girl (virtual health care company) and GI On DEMAND (virtual health care platform), CLL: None, SW: Consultant Boston Scientific, Medtronic, Cernostics, Interpace, Exact Sciences. Supported by NIH/NIDDK U34DK124174 and University of Colorado Department of Medicine Outstanding Early Scholars Award, SGP: Olympus America (research support), Freenome Inc. (research support), ERBE USA (honorarium).

\section{SUPPLEMENTARY MATERIALS}

Supplementary materials can be found via https://doi. org/10.15430/JCP.2021.26.4.298.

\section{ORCID}

Anjali Parekh, https://orcid.org/0000-0002-0291-9956

Camille J. Hochheimer, https://orcid.org/0000-0002-0984-0909 Jeannine M. Espinoza, https://orcid.org/0000-0002-7453-2400 Jordan J. Karlitz, https://orcid.org/0000-0001-5686-7365

Carmen L. Lewis, https://orcid.org/0000-0002-9739-2850

Sachin Wani, https://orcid.org/0000-0002-5150-3623

Swati G. Patel, https://orcid.org/0000-0002-1035-4924

\section{REFERENCES}

1. Siegel RL, Jakubowski CD, Fedewa SA, Davis A, Azad NS. Colorectal cancer in the young: epidemiology, prevention, management. Am Soc Clin Oncol Educ Book 2020;40:1-14.

2. Siegel RL, Miller KD, Goding Sauer A, Fedewa SA, Butterly LF, Anderson JC, et al. Colorectal cancer statistics, 2020. CA Cancer J Clin 2020;70:145-64.

3. Siegel RL, Miller KD, Jemal A. Colorectal cancer mortality rates in adults aged 20 to 54 years in the United States, 1970-2014. JAMA 2017;318:572-4.

4. Patel SG, Boland CR. Colorectal cancer in persons under age 50: seeking causes and solutions. Gastrointest Endosc Clin N Am 2020;30:441-55.

5. Patel SG, Murphy CC, Lieu CH, Hampel H. Early age onset colorectal cancer. Adv Cancer Res 2021;151:1-37.

6. Rex DK, Boland CR, Dominitz JA, Giardiello FM, Johnson DA, Kaltenbach $\mathrm{T}$, et al. Colorectal cancer screening: recommendations for physicians and patients from the U.S. MultiSociety Task Force on Colorectal Cancer. Gastroenterology 2017;153:307-23.

7. Boardman LA, Vilar E, You YN, Samadder J. AGA clinical practice update on young adult-onset colorectal cancer diagnosis and management: expert review. Clin Gastroenterol Hepatol 2020;18:2415-24.

8. Rex DK, Johnson DA, Anderson JC, Schoenfeld PS, Burke CA, Inadomi JM. American College of Gastroenterology guidelines for 
colorectal cancer screening 2009 [corrected]. Am J Gastroenterol 2009;104:739-50.

9. Wolf AMD, Fontham ETH, Church TR, Flowers CR, Guerra CE, LaMonte SJ, et al. Colorectal cancer screening for average-risk adults: 2018 guideline update from the American Cancer Society. CA Cancer J Clin 2018;68:250-81.

10. US Preventive Services Task Force, Davidson KW, Barry MJ, Mangione CM, Cabana M, Caughey AB, et al. Screening for colorectal cancer: US Preventive Services Task Force recommendation statement. JAMA 2021;325:1965-77.

11. Patel SG, May FP, Anderson JC, Burke CA, Dominitz JA, Gross $\mathrm{SA}$, et al. Updates on age to start and stop colorectal cancer screening: recommendations from the U.S. Multi-Society Task Force on Colorectal Cancer. Gastroenterology 2022;162:285-99.

12. Shaukat A, Kahi CJ, Burke CA, Rabeneck L, Sauer BG, Rex DK. ACG clinical guidelines: colorectal cancer screening 2021. Am J
Gastroenterol 2021;116:458-79.

13. NCCN. Colorectal cancer screening guidelines. https://www. nccn.org/professionals/physician_gls/pdf/colorectal_screening. pdf. Accessed May 1, 2021.

14. Sireci SG. The construct of content validity. Soc Indic Res 1998;45:83-117.

15. Lowery JT, Ahnen DJ, Schroy PC 3rd, Hampel H, Baxter N, Boland $\mathrm{CR}$, et al. Understanding the contribution of family history to colorectal cancer risk and its clinical implications: a state-ofthe-science review. Cancer 2016;122:2633-45.

16. Samadder NJ, Smith KR, Hanson H, Pimentel R, Wong J, Boucher $\mathrm{K}$, et al. Increased risk of colorectal cancer among family members of all ages, regardless of age of index case at diagnosis. Clin Gastroenterol Hepatol 2015;13:2305-11.e1-2.

17. Kellerman SE, Herold J. Physician response to surveys. A review of the literature. Am J Prev Med 2001;20:61-7. 